\title{
Orbit of Comet C/1850 Q1 (Bond)
}

\author{
Richard L. Branham Jr \\ Instituto Argentino de Glaciología y Nivología (IANIGLA), \\ C.C. 330,5500 Mendoza, Argentina \\ rlb@lanet.com.ar \\ Received 2002 May 2, accepted 2003 March 25
}

\begin{abstract}
Comet C/1850 Q1 (Bond) is one of a number of comets catalogued with parabolic orbits. Given that there are sufficient observations, 104 in right ascension and 103 in declination, it proves possible to calculate a better orbit. Some of the difficulties of working with 19th century observations, which show considerable scatter, are discussed. Rectangular coordinates, both of the comet and the Sun, are interpolated by a recursive version of Aitken's method, rendering unnecessary the need to specify an order for the interpolation. Comet Bond's orbit is slightly hyperbolic.
\end{abstract}

Keywords: celestial mechanics — comets: individual (C/1850 Q1) — methods: data analysis

\section{Introduction}

Many comets observed during the 19th century are still catalogued with parabolic orbits. This may be understandable given that, for long period comets at least, a parabola gives an acceptable first approximation to the orbit and is easier to calculate than an elliptic or hyperbolic orbit. But some of these comets have good observational histories, implying that we may be able to do better than a parabolic orbit (may because one must show that, given the errors of 19th century observations, a more refined orbit is statistically superior to a parabolic orbit). Apparently orbit calculators never got around to calculating a definitive orbit. But why should it be of interest to improve a parabolic orbit? What do we gain from doing so, particularly if the orbit is hyperbolic or of such large eccentricity that the comet, for all practical purposes, is gone forever? Then, who cares? To these questions three answers may be given. The first answer addresses craftsmanship. It is aesthetically displeasing to leave an orbit in a sloppy state when better can be done. The second is more practical in this age of interest in Near Earth Objects. Suppose the orbit turns out to be elliptical, albeit of high eccentricity? Suppose the comet returns within a few score years? Suppose that it passes close to the Earth? Suppose that the comet may impact with the Earth? Then we would regret having left the orbit in such a sorry state. Finally, good orbits are necessary for statistical analyses of comet orbits, essential for a study of the origin and evolution of comets.

Comet C/1850 Q1 (Bond) falls into this group of comets with parabolic but we can do better orbits. The literature disclosed 104 observations in right ascension $(\alpha)$ and 103 in declination $(\delta)$, implying that we can indeed do better than a parabolic orbit. Why start with Comet Bond rather than some other? George Bond discovered the comet on 29 August 1850 with the 15 in refractor of the Harvard College Observatory, the same refractor that William Bond, George's father, used that year to obtain the first clear daguerreotype of the moon (previous attempts had given blurry images). Thus, to calculate an orbit for Comet Bond honours, in some sense, an important milestone in astronomical history.

\section{Treatment of the Observations and Ephemerides}

I conducted a literature search of the journals published in the 19th century that include comet observations and also annual reports of some of the major observatories. Observations of Comet Bond were found in The Astronomical Journal, Monthly Notices RAS, and Astronomische Nachrichten. Table 1 shows the observatories and the number of observations made at each. The first observation, 29 August, and the last, 13 November, were made at the Harvard College Observatory. Figure 1 shows the observations. Observations were reduced to the common format of Julian Day (JD), referred to Terrestrial Time (TT), right ascension, and declination. This was necessary because some of the observers used north polar distance instead of $\delta$, some expressed $\alpha$ in degrees, minutes, and seconds rather than hours, minutes, and seconds, most, with the exception of the English observers, used mean time of place rather than Greenwich for the time of the observations, and the Dorpat observers recorded the time as sidereal rather than mean. The observations were all made with equatorial telescopes and filar or ring micrometers, the comet measured with respect to a nearby reference star. The comet's position was sometimes published as merely a mean or apparent place, but frequently the observer also published the reference star and sometimes even the difference in $\alpha$ and in $\delta$ from the reference star, $\Delta \alpha$ and $\Delta \delta$. Given that modern star catalogues are more precise than 19th century catalogues, it is more accurate when a definite reference star is mentioned to recalculate its apparent position, using the algorithm in Kaplan et al. (1989), from a recent modern catalogue, Tycho-2 (Høg et al. 2000), and apply $\Delta \alpha$ and $\Delta \delta$, corrected for differential aberration, 
Table 1. Observatories and observations with sources

\begin{tabular}{|c|c|c|c|}
\hline Observatory & $\begin{array}{l}\text { Obsns. } \\
\text { in } \alpha\end{array}$ & $\begin{array}{l}\text { Obsns. } \\
\text { in } \delta\end{array}$ & Reference $^{1}$ \\
\hline Kremsmünster, Austria & 10 & 10 & $\begin{array}{l}\text { AN, } 1850 \text {, No. } 741 \text {, } \\
\text { pp. } 329-30\end{array}$ \\
\hline Copenhagen, Denmark & 3 & 3 & $\begin{array}{l}\text { AN, } 1850, \text { No. } 742 \text {, } \\
\text { pp. } 347-48\end{array}$ \\
\hline Cambridge, England & 2 & 2 & $\begin{array}{l}\text { AN, } 1850, \text { No. } 739 \text {, } \\
\text { pp. } 299-300\end{array}$ \\
\hline Durham, England & 3 & 3 & MN, 1850 , Vol. 11, p. 13 \\
\hline Liverpool, England & 2 & 2 & $\begin{array}{l}\text { AN, } 1850, \text { No. } 734, \\
\text { pp. } 213-14\end{array}$ \\
\hline Marseilles, France & 19 & 18 & $\begin{array}{l}\text { AN, } 1850, \text { No. } 738, \\
\text { pp. } 285-86\end{array}$ \\
\hline Paris, France & 8 & 8 & $\begin{array}{l}\text { AN, } 1850, \text { No. } 734 \text {, } \\
\text { pp. } 219-20\end{array}$ \\
\hline Berlin, Germany & 1 & 1 & $\begin{array}{l}\text { AN, } 1850, \text { No. } 740 \text {, } \\
\text { pp. } 309-10\end{array}$ \\
\hline Bonn, Germany & 3 & 3 & $\begin{array}{l}\text { AN, } 1850, \text { No. } 738, \\
\text { pp. } 277-78\end{array}$ \\
\hline Hamburg, Germany & 7 & 7 & $\begin{array}{l}\text { AN, } 1850, \text { No. } 734, \\
\text { pp. } 211-12 \\
\text { AJ, } 1850 \text {, Vol. 1, p. } 154\end{array}$ \\
\hline Leipzig, Germany & 1 & 1 & $\begin{array}{l}\text { AN, } 1850, \text { No. } 738, \\
\text { pp. } 275-76\end{array}$ \\
\hline Markree Castle, Ireland & 2 & 2 & $\begin{array}{l}\text { AN, } 1850, \text { No. } 734 \text {, } \\
\text { pp. } 13-14\end{array}$ \\
\hline Dorpat, Russia & 6 & 6 & $\begin{array}{l}\text { AN, } 1850, \text { No. } 735 \text {, } \\
\text { pp. } 229-30\end{array}$ \\
\hline Geneva, Switzerland & 5 & 5 & $\begin{array}{l}\text { AN, } 1850, \text { No. } 734 \text {, } \\
\text { pp. } 211-12\end{array}$ \\
\hline Cambridge, USA & 18 & 18 & $\begin{array}{l}\text { AJ, } 1850 \text {, Vol. 1, } \\
\text { pp. } 141,154\end{array}$ \\
\hline New Haven, USA & 4 & 4 & AJ, 1850, Vol. 1, p. 156 \\
\hline (Old) US Naval, USA & 10 & 10 & AJ, 1850, Vol. 1, p. 140 \\
\hline
\end{tabular}

${ }^{1}$ AJ: Astronomical Journal; AN: Astronomishe Nachrichten; MN: Monthly Notices RAS

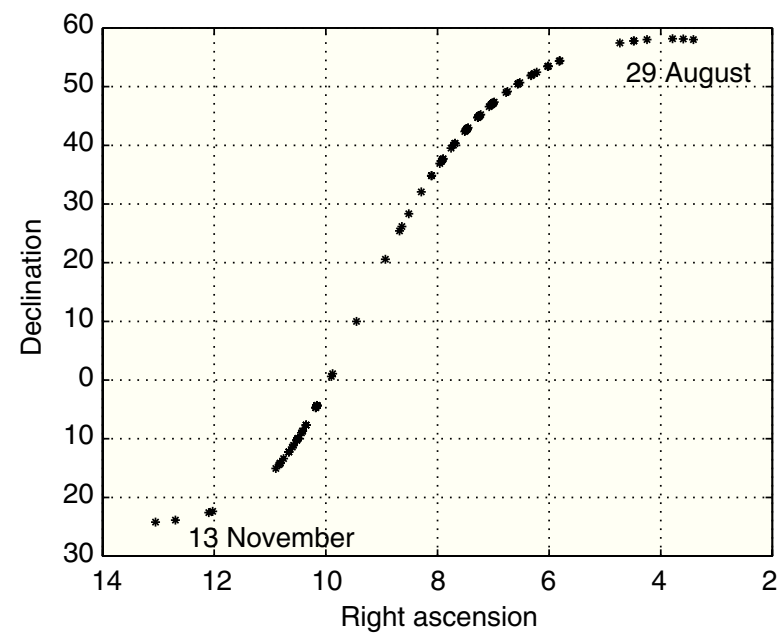

Figure 1 The observations.

to the new position. If $\Delta \alpha$ and $\Delta \delta$ were not given, the differences in the positions between the older catalogue and Tycho-2 were applied to the published positions of the comet. Given that the stated precision of the proper motions in the Tycho- 2 catalogue is less than $0.5 \mathrm{mas} \mathrm{yr}^{-1}$, even after 150 years the error in a star position should be under $0^{\prime \prime} .1$, much better than the errors of 19 th century catalogues.

The rectangular coordinates and velocities of the comet and the Earth were calculated by a program, used in numerous investigations previously, that treats the solar system as an n-body problem. The program is a 12 th order Lagrangian predictor-corrector that incorporates relativity by a Schwarzschild harmonic metric. To obtain coordinates and velocities for the Earth, the moon is carried as a separate body. This means a small step-size, $0^{d} .25$. To correct the comet's orbit partial derivatives are calculated by Moulton's method (Herget 1968), which integrates the partial derivatives to correct for the osculating rectangular coordinates and velocities at epoch JD2397020.5 along with the coordinates and velocities. The rectangular coordinates, after interpolation to the moment of observation for the Earth and to the moment of observation antedated by the light time correction to allow for planetary aberration, are then converted to a unit vector that is transformed to a mean or apparent place in $\alpha$ and $\delta$ by application of precession, nutation, annual aberration, relativity, and so forth. Because we are dealing with 19th century observations it is necessary to correct for the $\mathrm{E}$ terms of the aberration during the calculation of a mean place. The final step calculates an observed minus a computed place, $(\mathrm{O}-\mathrm{C})$, in $\alpha$ and $\delta$. To interpolate coordinates and partial derivatives I developed a more flexible procedure, recursive Aitken interpolation explained in the next section, that obviates the need to specify an order for the interpolating polynomial; one merely specifies the desired error for the interpolated quantities. Although there is mention of recursive interpolation in the literature, I have yet to find a specific algorithm. The Web site www.netlib.org, for example, a veritable treasure trove of numerical algorithms, has nothing for recursive interpolation.

\section{Recursive Aitken Interpolation}

To interpolate coordinates one is given a series of values for the arguments, $x_{0}, x_{1}, \ldots, x_{n}$, and corresponding function values, $f\left(x_{0}\right), f\left(x_{1}\right), \ldots, f\left(x_{n}\right)$. With ordinary interpolation one then specifies the order of the polynomial and the argument to be interpolated. The routine used, whether Lagrange, Bessel, Aitken, or some other, returns the interpolated function value and an estimate of its error. If $h$ is the interval of tabulation the formal error for a given order $n, E_{n}$, is given by $h^{(n+1)} f^{(n+1)}(\xi) /(n+1)$ !, where $\xi$ is some unknown value for $x$ between $x_{0}$ and $x_{n}$. This error represents what all polynomial interpolation methods give. If $f(x)$ is sufficiently smooth, then its higher derivatives do not oscillate violently and $E_{n}$ depends on $h$ and $n$; a higher order interpolation should give smaller error. If $f(x)$ or its higher derivatives are not smooth, then higher order does not imply greater precision in the interpolation. 
Aitken's method uses an array of coefficients $P_{i, j}$ defined as

$$
\begin{gathered}
P_{i, 0}=f\left(x_{i}\right), \quad i=0,1, \ldots, n, \\
P_{i, k+1}=\left[\left(x-x_{k}\right) P_{i, k}-\left(x-x_{i}\right) P_{k, k}\right] /\left(x_{i}-x_{k}\right), \\
i=0,1, \ldots n, \quad k=0,1, \ldots, i-1,
\end{gathered}
$$

where $f\left(x_{i}\right)$ refers to the data points, usually equally spaced although equal spacing is not required, and $x$ to the value to be interpolated. Equation (1) generates a lower triangular array of coefficients

$$
\begin{array}{ccccc}
P_{0,0} & & & & \\
P_{1,0} & P_{1,1} & & & \\
P_{2,0} & P_{2,1} & P_{2,2} & & \\
\vdots & & & & \\
P_{n, 0} & P_{n, 1} & P_{n, 2} & \cdots & P_{n, n}
\end{array}
$$

the last coefficient of which, $P_{n, n}$, interpolates the value, and the difference of the $n$th and $(n-1)$ th interpolated values, $P_{n, n}-P_{n-1, n-1}$, estimates the error. To use Aitken's method one stipulates the order of the interpolation and looks at the estimated error. If the latter is unacceptable, the order is increased. This implementation of Aitken's method is usually referred to as iteration because, according to equation (1), the $P$ s are generated by iterating from $P_{0,0}$ to $P_{n, n}$.

But a glance at equation (1) shows that use of recursion permits reversing the process: if the coefficients $P_{0,0}$, $P_{1,1}, \ldots, P_{n, n}$ are generated recursively, one can first specify the error desired and generate the coefficients recursively until $P_{n, n}-P_{n-1, n-1}$ satisfies the criterion. Modern languages such as Fortran 90, unlike earlier versions of Fortran, and $\mathrm{C} / \mathrm{C}++$ permit recursion. Suppose that we wish to interpolate planetary coordinates and velocities from an ephemeris tabulated at a one-day interval. A Fortran array $y(i, j)$, defined as a global variable in a module 'global', contains the data, where $i$ indexes the date and $j$ the coordinate or velocity we wish to interpolate. Table 2 shows possible Fortran 90 code for the polynomials.
How do the two techniques, iteration and recursion, compare regarding workload? From equation (1) the calculation of each $P$ involves, aside from the $P_{i, 0}$ values, only an assignment, two multiplications, one division, and four subtractions, or seven arithmetic operations per $P$. (This assumes that each arithmetic operation consumes the same clock cycles. On machines for which multiplications and divisions are slower than additions and subtractions, the count per $P$ becomes three.) From equation (1) the iterative technique requires

$$
\sum_{i=0}^{n} \sum_{k=0}^{i-1} 7=7 n(n+1) / 2
$$

operations to generate the lower triangular matrix of $P$ s. The recursive technique uses the same number of arithmetic operations per $P$, but each recursive call generates two more, plus the overhead of placing values on the stack. Thus, the operation count becomes

$$
7 \sum_{i=1}^{n} 2^{i}=7\left(2^{n+1}-2\right) .
$$

The ratio of recursion to iteration may be expressed as $f(n)=4\left(2^{n}-1\right) / n(n+1)$. For various values of $n$ we find

\begin{tabular}{rr}
\hline$n$ & $f(n)$ \\
\hline 8 & 14 \\
10 & 37 \\
12 & 105 \\
14 & 312 \\
16 & 964 \\
18 & 3066 \\
20 & 9986 \\
\hline
\end{tabular}

Thus, the expense of using recursion over iteration increases dramatically as the order increases. With the speed of modern computers computational efficiency is really a secondary concern unless the depth of the recursion becomes excessive or the stack becomes exhausted and aborts the program. It is, therefore, a good idea to

Table 2. Fortran 90 code for recursive Aitken interpolation 
specify a limit for the order of the recursive calls to handle pathological functions that give poor results regardless of the order used, such as functions with violently oscillating higher derivatives. Recursion's main advantage lies in permitting automatic control over the order of the interpolation. For the interpolation of the coordinates and velocities for Comet Bond the order always started at eight, but frequently increased to nine or sometimes ten. The user need not concern himself with establishing an order for the interpolation and then checking if it is adequate. In this sense recursive interpolation becomes analogous to automatic control of the interval with numerical integration of differential equations or numerical quadrature.

\section{The Observations}

After the first differential corrections, corresponding to orbits with large $(\mathrm{O}-\mathrm{C}) \mathrm{s}$, it became evident that the $(\mathrm{O}-\mathrm{C}) \mathrm{s}$ and post-fit residuals nevertheless still embodied some large errors; the mean error of unit weight $\sigma(1)$ from the final solution is $5^{\prime \prime} .16$. This is higher than the $3^{\prime \prime} .62$ mean error from observations made of minor planets in 1847-1849 in a previous study of mine (Branham 1991). A number of reasons explain this. The comparison stars were taken from catalogues that have large errors compared with modern catalogues. Lalande's Histoire Celeste Francoise, as updated by Baily, used by Valz at Marseilles, sometimes shows errors over $1^{s}$ in $\alpha$ and $20^{\prime \prime}$ in $\delta$ compared with Tycho-2. Fortunately, Valz also published his observations as $\Delta \alpha$ and $\Delta \delta$ as well as apparent places and they could thus be referred to places calculated from Tycho-2. But this was not always possible, and one had to take the apparent or mean place as given by the observer. But even differential observations referred to an apparent place calculated from Tycho- 2 contain some large errors because the comet itself was frequently difficult to observe. Typical observers' comments are: 'comet faint'; 'very diffused and faint nebulosity...no nucleus'; 'weak, round nebulosity, without noticeable nucleus or coma'. Micrometric observations of such an object will not be easy. Not only the comet itself presented problems, but the observations themselves were often made at large zenith distances (median zenith distance is $72^{\circ}$ ). One observer mentions specifically that the comet was measured when very close to the horizon. (There was, however, no significant correlation between the zenith distance and the size of the residual: the correlation coefficient was equal to -0.049 .) There were also numerous clerical errors. One $\Delta \delta$ was published with the wrong sign, and at least five of the reference stars were incorrect. This type of error could only be found by detailed detective work. The wrong reference star, for example, could sometimes be detected by taking an abnormally large $(\mathrm{O}-\mathrm{C})$ and seeing if there was a relatively bright star near the published reference star that would reduce the $(\mathrm{O}-\mathrm{C})$ to something acceptable. But some clerical errors may nevertheless remain undetected.

But given that the data are noisy, one must consider ways of assigning higher weight to the better observations.

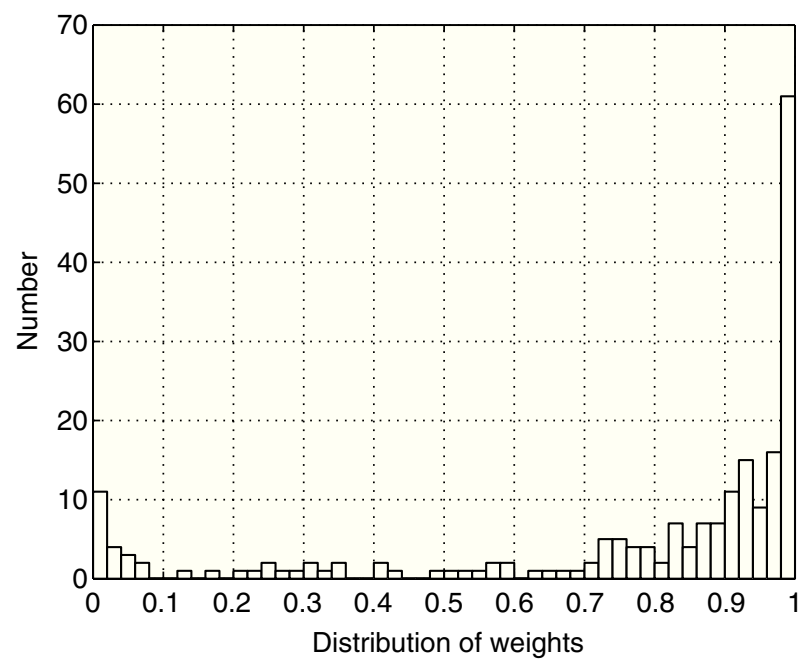

Figure 2 Distribution of weights from the biweight.

Differential observations referred to an apparent place calculated from Tycho- 2 should be better than apparent places published by the observer. Weighting, however, is tricky and often degenerates into an exercise into one's visceral feeling as to the quality of the observation. To achieve some objectivity in such a subjective endeavour I decided to use a weighting scheme that assumes nothing as to the quality of a given type of observation, but rather assigns weight based on the magnitude of the postfit residual. This type of weighting is common in iteratively reweighted least squares (Branham 1990). I decided to use the weighting known as 'biweight' because it gave good results in a previous study of mine (Branham 1986). One scales the post-fit residual $r_{i}$ by the median of the residuals and assigns a weight $w t$ as

$$
\begin{aligned}
& w t=\left[1-\left(r_{i} / 4.685\right)^{2}\right]^{2} ; \quad r_{i} \leq 4.685 \\
& w t=0 ; \quad r_{i}>4.685 .
\end{aligned}
$$

Rather than start from a least squares solution, I decided to use the robust $\mathrm{L}_{1}$ criterion (Branham 1990) for the first approximation, calculate the residuals from this solution, compute the weights, and then calculate a least squares solution. Because the first approximation is good, it becomes unnecessary to iterate the solutions. Figure 2 shows the distribution of the weights. The median weight was $0.91 ; 82.1 \%$ of the observations received weights greater than $0.5,76.8 \%$ weights between 0.7 and 1 , and $67.2 \%$ weights between 0.8 and 1 . Only $9.7 \%$ of the weights were under 0.1 , including 11 zero weights. The biweight, therefore, eliminated 11 residuals, $5.3 \%$ of the total, modest but not excessive trimming.

\section{The Solution}

Table 3 shows the final solution for the rectangular coordinates, $x_{0}, y_{0}, z_{0}$, and velocities, $\dot{x}_{0}, \dot{y}_{0}, \dot{z}_{0}$, along with their mean errors. Table 4 exhibits the covariance and correlation matrices for the solution. The correlations are high, but the solution itself is stable because a singular 
Table 3. Solution for rectangular coordinates and velocities

\begin{tabular}{lcl}
\hline Unknown & Value & Mean Error \\
\hline$x_{0}$ & 0.800062585008864 & 0.000015116006889 \\
$y_{0}$ & 0.254178352285609 & 0.000022025333264 \\
$z_{0}$ & 0.202299258909604 & 0.000017377821954 \\
$\dot{x}_{0}$ & -0.018968619113946 & 0.000001491359962 \\
$\dot{y}_{0}$ & 0.015197473728116 & 0.000000205744985 \\
$\dot{z}_{0}$ & -0.009745969276271 & 0.000001242258420 \\
$\sigma(1)$ & $5^{\prime \prime} .16$ & \\
\hline
\end{tabular}

Table 4. Covariance (upper triangle) and correlation (lower triangle) matrices

\begin{tabular}{rrrrrr}
\hline 0.2869 & -0.4137 & -0.3236 & 0.0280 & 0.0010 & 0.0230 \\
-0.9896 & 0.6091 & 0.4767 & -0.0404 & -0.0019 & -0.0339 \\
-0.9810 & 0.9919 & 0.3792 & -0.0315 & -0.0017 & -0.0266 \\
0.9889 & -0.9797 & -0.9675 & 0.0028 & 0.0001 & 0.0023 \\
0.2659 & -0.3313 & -0.3689 & 0.2172 & 0.0001 & 0.0001 \\
0.9775 & -0.9858 & -0.9805 & 0.9686 & 0.3558 & 0.0019 \\
\hline
\end{tabular}

Table 5. Hyperbolic orbital elements and mean errors

\begin{tabular}{lcl}
\hline Unknown & Value & Mean Error \\
\hline$T_{0}$ & JD2397050.32539 & $0^{d} .00683$ \\
& 19.82539 Oct. 1850 & \\
$a$ & -923.723665704311 & 201.508457295895 \\
$e$ & 1.00061230501936 & 0.00013373819465 \\
$q$ & 0.565600637009132 & 0.016286429624161 \\
$\Omega$ & $234^{\circ} .492067975171$ & $0^{\circ} .005157521916$ \\
$i$ & $21^{\circ} .8798895958628$ & $0^{\circ} .0015069065954$ \\
$\omega$ & $69^{\circ} .1265933409261$ & $0^{\circ} .1965992486476$ \\
\hline
\end{tabular}

value decomposition shows that the condition number of the matrix of the equations of condition is 261 , hardly excessive.

Table 5 gives the orbital elements corresponding to the rectangular coordinates of Table 3: the time of perihelion passage, $T_{0}$; the eccentricity, $e$; the semi-major axis (negative for hyperbolic orbits), $a$; perihelion distance, $q$; the inclination, $i$; the node, $\Omega$; and the argument of perihelion, $\omega$. The orbit itself is hyperbolic, although the mean error of $e$ indicates that the possibility of a highly eccentric ellipse cannot be excluded. The calculation of the mean errors of the orbital elements is onerous and exhibits one of the weaknesses of Moulton's method, at least when used to correct rectangular coordinates and velocities. To calculate the orbital elements themselves from the rectangular coordinates and velocities is straightforward, but the mean errors have to be computed by use of the elements of the covariance matrix and the partial derivatives of the orbital elements with respect to the coordinates and velocities, $\partial e / \partial x_{0}$ for example; see Rice (1902) for a detailed discussion. Because the orbital elements are related to the coordinates and velocities in a highly transcendental manner, the calculation of these partial derivatives is extremely tedious.

Figure 3 shows the residuals from the final solution. Although they exhibit considerable scatter - the median

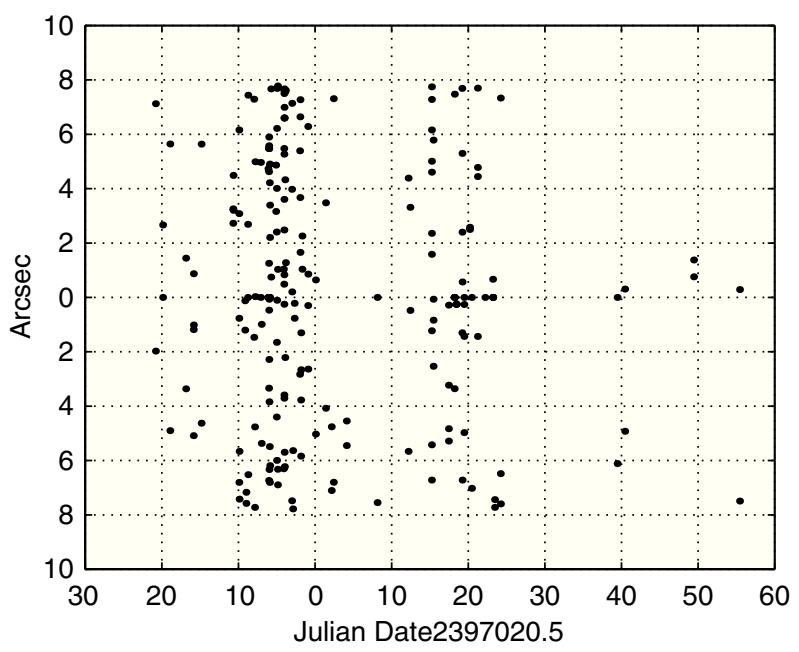

Figure 3 Residuals from the final solution.

absolute value for the residuals is $4^{\prime \prime} .54$ - they are random. A runs test for randomness shows 106 runs out of an expected 103.5 with a standard deviation of 7.1. Nor does spectral analysis of the residuals reveal any periodicities. Random, but not normal. The residuals are slightly skewed, factor of skewness $=0.063$, and platykurtic, kurtosis $=-0.909$. But given that they are random one can consider the solution acceptable.

Is the new orbit better than the parabolic orbit initially used? The answer is a definite yes. The ratio of the sum of the squares of the pre-fit $(\mathrm{O}-\mathrm{C})$ s between the initial orbit and the final orbit, before treating the observations in any way such as eliminating outliers, is 382 . By any statistical test one cares to use this represents significant improvement.

\section{Conclusions}

One hundred and four observations in $\alpha$ and 103 in $\delta$, made between 29 August and 13 November 1850, are used to calculate an orbit for Comet C/1850 Q1 (Bond). The orbit is slightly hyperbolic.

\section{Acknowledgments}

The literature search for this paper was conducted during a visit to the US Naval Observatory. I thank Brenda Corbin, librarian of the Observatory, and Gregory Shelton, assistant librarian, for their help and cordiality.

\section{References}

Branham Jr, R. L. 1986, QJRAS, 27, 182

Branham Jr, R. L. 1990, Scientific Data Analysis (New York: Springer), Section 5.5, Chapter 6

Branham Jr, R. L. 1991, in Mathématiques Appliquées aux Sciences de L'Ingénieur, eds. C. Carasso, C. Conca, R. Correa, \& J. -P. Puel (Toulouse: Cépaduès), 81

Herget, P. 1968, AJ, 73, 737

Høg, E., et al. 2000, The Tycho-2 Catalogue (Washington, DC: US Naval Observatory)

Kaplan, G. H., Hughes, J. A., Seidelmann, P. K., \& Smith, C. A. 1989, AJ, 97, 1197

Rice, H. L. 1902, AJ, 22, 149 